\title{
Drogas en la Leche Materna
}

\author{
Dr. Alberto Duarte-Contreras*
}

Se adelanta una campaña universal en pro de la alimentación natural o materna, basada en razones fisiológicas, demográficas y económicas. Ello explica la preocupación de obstetras y pediatras por conocer la veracidad de tantas creencias populares sobre los efectos que pueden producir al lactante diferentes dietas y drogas ingeridas por las madres que amamantan.

Debemos admitir que la información farmacológica sobre medicamentos y sustancias químicas contenidas en la leche materna es sorprendentemente incompleta y fragmentaria, y que son pocos los estudios sistemáticos y amplios que se han adelantado sobre este tema en seres humanos. Con todo, el especialista y el médico familiar deben tener en mente algunos principios generales y ciertas precauciones especiales. Cualquier tratamiento farmacológico suministrado a una madre que lacta debe ser beneficioso para ella sin que vaya a ser perjudicial para el lactante.

Ciertas drogas administradas a la madre durante el embarazo pueden tener efectos adversos sobre el feto (15).

De la misma manera un buen número de componentes farmacológicos administrados a la madre en el período de lactancia, se excretan por la leche y,

* Del Departamento Gíneco Obstétrico del Hospital San Juan de Dios. Cúcuta, Colombia. al ser ingeridos por el niño, pueden producirle efectos nulos, benéficos o perjudiciales.

La leche se sintetiza por acción de la prolactina en las células secretoras o alveolares del tejido mamario y éstas la vierten hacia los alvéolos. Merced a la acción contráctil que poseen las células mioepiteliales perialveolares, y bajo el influjo de la ocitocina, se verifica la eyección de la leche hacia el sistema de conductos. (Figuras 1 y 2).

La leche se compone primordialmente de proteínas, carbohidratos y grasas. Las proteínas se reúnen en los ribosomas del retículo endoplásmico de las células alveolares, pasan al aparato de Golgi y allí reciben el complemento de carbohidratos para constituir las glucoproteínas; éstas se almacenan en vacuolas para ser expulsadas luego a la luz de los acinos. La lactosa se sintetiza en el aparato de Golgi por acción de la lactosa-sintetasa, una enzima constituida por dos proteínas, A y B o lactoglobulina alfa. Las grasas se encuentran en forma de gotas envueltas en una membrana compleja y por el fenómeno de pinocitosis invertida se deslizan hacia la luz acinal.

La excreción de los fármacos, componentes de peso molecular bajo, se hace por diferentes mecanismos: transporte activo, difusión pasiva, o por unión de las moléculas a las vesículas que contienen proteínas para verter con ellas a la luz alveolar. 
Lactación: Secreción láctea - esteroides corticosuprarrenales y prolactina.
En el niño: control

endocrino no específico

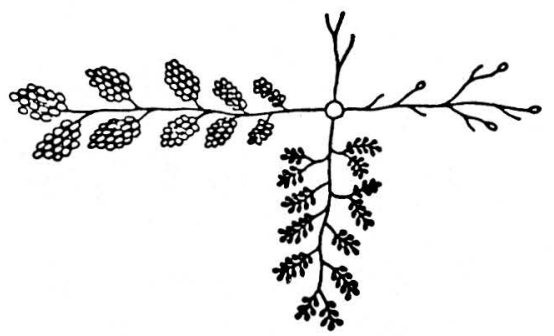

Embarazo: crecimiento de la región lóbulo-alveolar - estrógenos, progesterona; esteroides corticosuprarrenales, prolactina ( = hormona de crecimiento).
En la pubertad: crecimiento

y brote de los canales. estrógenos, esteroides corticosuprarrenales $y$ hormona de crecimiento.

FIGURA N: 1 - Control endocrino de la glándula mamaria antes de la pubertad, en la pubertad, durante el embarazo y durante la lactación. (Reproducido de Anales Nestlé, 129).

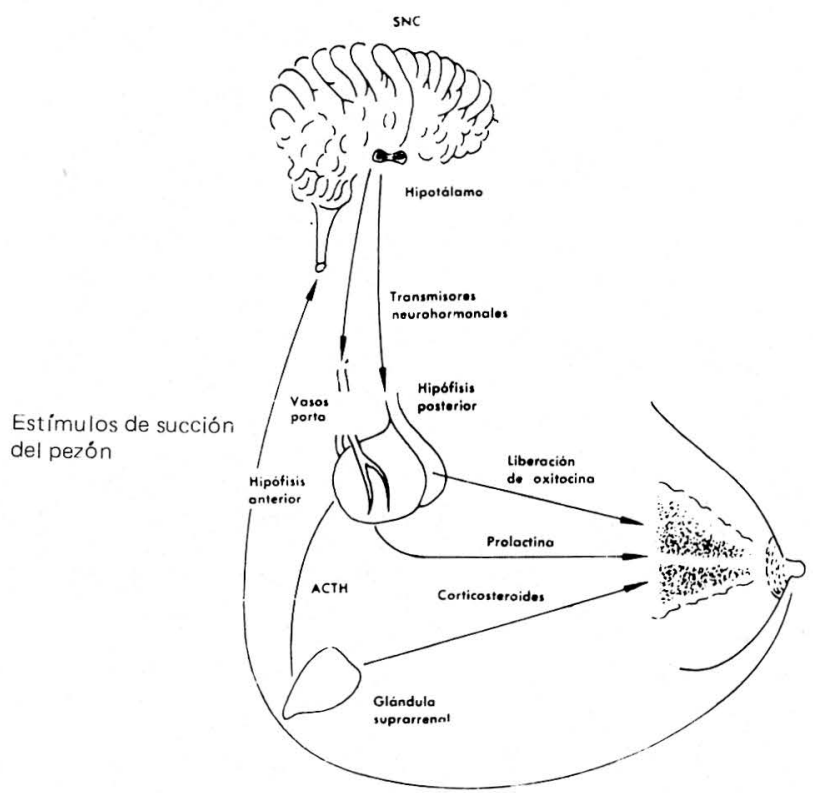

FIGURA No 2 - Factores neuro-endocrinos que intervienen en el mantenimiento de la lactación. 
El pH de la leche es ligeramente inferior al $\mathrm{pH}$ del plasma; por ello las bases débiles tienden a presentar un cociente leche/plasma alto, o sea concentraciones en la leche iguales o superiores a las del plasma. Los ácidos débiles presentan un cociente leche/plasma bajo, o sea concentraciones en la leche iguales 0 inferiores a las del plasma.

En la leche materna aparecen numerosos compuestos extraños que lógicamente son ingeridos por el lactante. Cualquier fármaco que se encuentre circulando en el organismo materno puede traspasar el endotelio capilar e ir a las células alveolares para ser secretado luego con la leche. La mayoría de los medicamentos ingeridos por la madre se encuentran en la leche materna por lo general en concentraciones muy bajas, concentraciones que dependen de factores tales como la cantidad circulante en la sangre de la mujer lactante, la liposolubilidad del compuesto y su grado de ionización o sea la biodisponibilidad de la droga en el organismo materna. Es importante, además, tener en cuenta la vía de administración del fármaco, pues de ello depende la concentración que pueda alcanzar en el plasma; tiene también importancia el tiempo que demore la droga entre su ingestión por la madre y el momento en que se pueda detectar en la leche.

El fármaco aparecerá más rápidamente cuando la administración se verifica por vía parenteral que por vía oral. Cuando la administración se verifica por vía oral se deben tener en cuenta algunas modalidades: la presentación del fármaco (tabletas, soluciones, de desintegración retardada), la ingestión previa de alimentos y la composición físico química. La elevada y prolongada concentración de un fármaco en la circulación materna no sólo aumenta la posibilidad de su paso a la leche sino también la mayor o menor concentración que logre alcanzar en ella.

Algunos fármacos se combinan con las grasas o con las proteínas de tos tejidos y pueden permanecer en ellos largo tiempo; si su administración es repetida pueden producir un efecto acumulativo. Las funciones hepática y renal, inmaduras en la primera infancia, pueden retardar la excreción o la inactivación de las drogas, y así la ingestión continua de leche materna puede ocasionar concentraciones clínicamente importantes de ciertos fármacos en la sangre del niño.

La presencia de drogas en la leche materna no siempre implica efectos perjudiciales para el lactante ya que este producto puede ser farmacológicamente inactivo, o puede ser destruido por el aparato gastrointestinal del niño, o puede que no sea absorbido. Debemos tener siempre en mente que pueden presentarse efectos indeseables mínimos, no demostrables, que lleguen luego a producir alteraciones en la omeostasis del lactante.

Algunos productos como el fenobarbital o el clordano (insecticida), aumentan o inhiben la actividad de los sistemas enzimáticos, aun cuando se desconocen los efectos de estas acciones a largo plazo. En la actualidad se adelantan estudios sobre si la cantidad de estos productos excretada por la leche influye sobre las enzimas microsómicas del lactante (12).

Hay lactantes que por anomalías genéticas heredadas pueden reaccionar fácil y rápidamente a la ingestión de fármacos a través de la leche materna, aun en dosis mínimas, insuficientes para producir de por sí efectos indeseables. Ciertas drogas administradas a la madre pueden alterar en el lactante determinadas funciones, facilitando asi los efectos nocivos de otros fármacos que se administran simultánea o posteriormente; por ejemplo al administrar anticoagulantes a la madre se puede alterar la función hemostática del lactante, y en el caso de que a este niño se le tormule aspirina de inmediato por un tiempo más o menos prolongado, puede hacer fácilmente hemorragias, severas en algunos casos. Otras drogas como el tiouracilo y el yodo se excretan por la leche en concentraciones superiores a las que tienen en el plasma 
materno, produciendo serios trastornos en las funciones normales del niño $(26)$.

Cuando se adelánta simultáneamente un tratamiento a madre e hijo con la misma droga, a dosis, terapéuticas, sin destetar al bebé, se puede presentar en el lactante una sobredosis farmacológica con reacciones de hipersensibilidad (42). Se pueden encontrar en la leche materna pequeñas cantidades de fármacos inofensivos que Ilegan a afectar al niño cuando éste es portador de defectos enzimáticos. Tal el caso de la "ictericia por leche materna" causada por inhibidores desconocidos; esta ictericia es la resultante de un trastorno de maduración de la glucuronil-transferasa. Como el intestino del recién nacido permite la absorción de macromoléculas no digeridas, el niño alimentado al seno se puede sensibilizar a mínimas cantidades de droga contenidas en la leche materna (51).

Cuando la madre que lacta presenta una disminución de la función renal pueden aparecer en la leche fármacos en concentraciones muy elevadas, que de otra manera se hallarían en mínima cantidad.

Teniendo presente que la maternidad es un todo y que la lactancia natural forma parte de ella, que "las madres que escogen la lactancia natural centran su interés en el bienestar del niño", se hace indispensable conocer qué efectos pueden tener sobre el bebé algunas sustancias administradas a la madre durante el período de lactancia. Es lo que nos proponemos en la presente comunicación, advirtiendo que en un trabajo como éste es imposible recopilar todas las drogas o sustancias que pueden ser ingeridas por la madre.

\section{Analgésicos}

PROPOXIFENO. (Darvón u otros). Se considera generalmente como un análogo de la Metadona aunque es un éster y no una cetona (32). Se excreta por la leche. El lactante puede recibir una cantidad farmacológicamente a ctiva cuando la madre lo ingiere a dosis máximas, pero se considera que un niño de 5.5 a 7.7 kilos de peso podría recibir, sin peligro alguno, hasta $1 \mathrm{mg}$. diario, dosis ésta francamente significativa (12).

SALICILATOS. ASPIRINA. Se les encuentra en la leche materna en cantidades moderadas, por lo que se considera improbable un efecto perjudicial para el lactante cuando la madre los ingiere de manera ocasional. No se ha estudiado su efecto cuando la ingestión se hace por tiempo prolongado y a dosis altas en el tratamiento, v.g. de la artritis reumatoidea, pero se cree que en esta contingencia pueda acarrear una tendencia hemorrágica interfiriendo con la función trombocítica del niño o disminuyendo la cantidad de protrombina en la sangre tal como sucede en los neonatos cuyas madres recibieron esta droga por tiempo prolongado durante la gestación, $(12,15)$.

\section{Anticoagulantes}

Usados preferencialmente para el tratamiento de las flebitis postparto. Algunos autores dicen que cuando los anticoagulantes alcanzan altos niveles en la leche materna se pueden observar hemorragias en los lactantes, algunas muy severas (27).

HEPARINA (Liquemine). No pasa a la leche materna.

\section{DISHIDROXICUMARINA} rol). Se administró a 125 mujeres que amamantaban sin que se hubiesen observado en los lactantes alteraciones en el tiempo de protrombina, ni hemorragias $(9)$.

FENIDIONA (Hedulin). Eckstein informó que un niño de cinco semanas, alimentado al seno, hizo un hematoma voluminoso en el sitio de intervención para tratamiento de una hernia inguinal; la madre tomaba Fenindiona. El niño presentó un tiempo de protrombina $50 \%$ del normal; el tiempo de protrombina de la madre bajó al $23 \%$ del normal después de suspendida la lactancia, lo que se consideró como una 
acumulación del fármaco en el organismo materno (16).

WARFARINA (Coumadin). Se detecta en cantidades insignificantes en la leche materna (10).

\section{Anticonvulsivos}

Empleados para el tratamiento de la epilepsia.

CARBAMAZEPINA (Tegretol). Se excreta en mínima cantidad por la leche materna (35).

DIFENILHIDANTOINA (Cumatil, Epamín, Heparina, Om-Hidantoína). Se ha usado de manera continua en el tratamiento de convulsiones y se ha demostrado que es mínima la cantidad que se excreta por la leche (33). Puede incrementar la actividad de las enzimas hepáticas.

FENITOINA (Dilantín). Se excreta en mínima cantidad por la leche materna y no se han observado alteraciones en los lactantes.

FENOBARBITAL. Tyson suministró $100 \mathrm{mg}$. diarios de Fenobarbital a 41 madres que lactaban niños menores de un año y sólo observó una discreta somnolencia en dos de ellos (48). La experiencia enseña que las madres que amamantan pueden recibir por largo tiempo dosis diarias anticonvulsivas hasta de $300 \mathrm{mg}$. sin que se afecte el bebé. Por lo demás, el fenobarbital es insustituible y bien tolerado en niños de pecho. En estudios realizados en animales se ha encontrado el fenobarbital en concentración suficientemente elevada como para aumentar la actividad de las enzimas hepáticas metabolizadoras del fármaco. Este aumento de actividad enzimática puede elevar en el lactante el metabolismo de otras drogas que esté tomando la madre, o el de los esteroides endógenos del niño, o causar otros efectos indeseables (19).

PRIMIDONA (Mysoline). En el hígado se metaboliza a fenobarbital. Como hay pacientes cuyos sistemas enzimáticos hepáticos transforman con rapidez y en gran cantidad la primidona en fenobarbital, la misma casa productora recomienda no lactar a las madres que estén tomando esta droga.

\section{Antihipertensivos}

GUANETIDINA (Ismelín). Se han dosificado mínimas cantidades en la leche materna pero no se ha constatado efecto alguno indeseable en los lactantes (46).

METILDOPA (Aldomet). No se conocen alteraciones en niños que lactan aun cuando la madre haya recibido este fármaco por tiempo prolongado.

PROPANOLOL (Inderal). Se excreta por la leche en cantidad tan insignificante que no puede producir efecto alguno indeseable en el lactante (2) .

RESERPINA. Se dosifica en la leche materna. La casa productora previene sobre posibles efectos indeseables al lactante, los mismos que se pueden observar en el recién nacido de madre que ha recibido dosis altas de reserpina al final del embarazo: letargo, diarrea, anorexia, cianosis, congestión nasal y secreciones del aparato respiratorio capaces de interferir con la respiración del niño $(12,15)$.

\section{Antimicrobianos}

Cualquier agente antimicrobiano que contenga la leche materna puede alterar la flora bacteriana intestinal del niño, flora que juega un papel preponderante en el desarrollo temprano del sistema inmunitario (51). Los quimioterapéuticos, una vez administrados a la madre pasan a la leche en pequeñas concentraciones (21). Por regla general no está contraindicada su administración a la madre que amamanta si bien se deben tener en cuenta algunas recientes informaciones.

ACIDO NALIDIXICO (Wintomylón). Se ha informado el hallazgo de anemia hemolítica en un lactante cuya madre tomaba ácido nalidíxico $(3,12)$. 
AMPICILINA. Existe la posibilidad de producir candidiasis y diarrea en los lactantes cuando se administran dosis altas y sostenidas a la madre por tiempo prolongado (55). La proporción entre la concentración en leche y plasma (L: P) es de 1: 5 a 10.

CLORANFENICOL. Solamente se han detectado cantidades muy pequeñas del fármaco en la leche materna, al parecer insuficientes para producir el "síndrome del niño gris". Con todo, se aconseja suspender la lactancia durante el tratamiento con cloranfenicol por el temor a que se produzca depresión de la médula ósea del lactante (11).

METRONIDAZOL (Flagyl) y TINIDAZOL (Fasigyn). Se excretan por la leche. Se les conşidera como carcinogenéticos en roedores y mutagénicos en bacterias $(17,40,50)$, por cuya razón la casa productora los contraindica para la mujer que amamanta, aun cuando se desconozca, aun si estos compuestos sean o no perjudiciales para el lactante.

NITROFURANTOINA (Furadantina, Macrodantina). Se han detectado mínimas cantidades en la leche materna. No producen discrasias sanguíneas.

Sin embargo, Versano informó que un lactante menor de un año, cuya madre tomaba furadantina, había presentado anemia hemolítica; este bebé tenía deficiencia de glucosa 6 fosfato deshidrogenasa G6PD (49).

PENICILINA. Se encuentra en la leche materna y puede aumentar en el niño el riesgo de reacciones de sensibilización posterior. L: $P$ es de 1: 5 a 10 lo mismo que en la Ampicilina.

SULFONAMIDAS. Se encuentran en la leche materna en muy baja cantidad. Con todo, se llama la atención sobre la posibilidad de que pueden causar anemia hemolítica en menores de un año portadores de deficiencia de glucosa 6 deshidrogenasa G6PD $(12,23)$. Podrían también aumentar el peligro del kernícterus en niños con incompatibilidad Rh o ABO.
TETRACICLINAS. Teóricamente pueden producir manchas de tipo marmóreo en los dientes en desarrollo del lactante. Es posible que las tetraciclinas no sean absorbidas por el lactante ya que forman complejos con el calcio de la leche; se ignora si por este mecanismo puedan producir hipocalcemia. (11). L: P puede ser del 20 al $90 \%$ de la concentración sérica.

\section{Antitiroideos}

TIOURACILO y PROPILTIOURACILO. Contraindican la lactancia. Alcanzan concentraciones más elevadas en la leche que en la sangre materna y pueden inhibir la función tiroidea del niño (55).

YODO RADIACTIVO. La madre que recibe dosis terapéuticas de yodo radiactivo no debe amamantar a su hijo. La cantidad de isótopos que se detectan en la leche materna son suficientes para destruir la glándula tiroides del lactante. Las mínimas cantidades de yodo utilizadas para practicar pruebas diagnósticas, como la captación de yodo radiactivo, pueden aumentar el riesgo de un cáncer tiroideo en etapas más avanzadas de la vida (7).

YODUROS. Pasan también a la leche materna si bien en pequeñas cantidades; sin embargo pueden producir hipotiroidismo o bocio en los lactantes (11) .

\section{Diuréticos}

Las casas productoras ordinariamente advierten en circulares adjuntas que se deben utilizar con prudencia en mujeres lactantes ya que pueden producir serias alteraciones electrolíticas en el niño cuando la madre las toma a dosis altas.

CLORTALIDONA (Higrotón). Mulley dice que las concentraciones encontradas en la leche materna son suficientes como para que no sea usado en la mujer que amamanta (34).

TIAZIDAS. Todas se pueden dosificar en la leche materna pero en cantidades tan reducidas que parece no puedan 
acarrear efecto alguno indeseable en el niño que lacta (52). Sin embargo se ha llamado la atención sobre la posibilidad de una trombocitopenia en lactantes cuyas madres tomaban clorotiazida (i1).

\section{Hormonas}

Nos interesan únicamente las hormonas que conservan su actividad biológica después de ser absorbidas por el aparato gastrointestinal del niño. Así, la insulina, la adrenalina y la corticotropina no nos preocupan pues se destruyen al pasar por el tubo gastrointestinal.

ANTICONCEPTIVOS ORALES. Se han estudiado las concentraciones de estas hormonas en la leche materna por diferentes métodos tales como técnicas biológicas, o la administración de noretinodrel marcado: los resultados han sido contradictorios $(28,39)$. No se han realizado y urge la elaboración de estudios adicionales sobre la excreción de anticonceptivos orales y sus metabolitos por la leche materna; como ocurre con la mayoría de las drogas no se han investigado los posibles efectos a largo plazo en niños amamantados durante varios meses cuyas madres tomaban los anticonceptivos. Curtis en 1964 informó que un niño de tres meses presentó ginecomastia al ingerir su madre una doble dosis de anticonceptivos; (14); desafortunadamente en este caso no se dosificaron las concentraciones hormonales en la leche. Para la práctica podemos decir que parece no se hayan comprobado efectos indeseables en niños alimentados con leche materna cuando la madre toma anticonceptivos. Recordemos que los anticonceptivos abrevian el tiempo de lactancia por inhibición paulatina de la secreción láctea. Estas mismas apreciaciones las podemos tener presentes en relación con los anticonceptivos hormonales administrados por vía parenteral.

CORTICOSTEROIDES. Se encuentran en la leche materna en baja concentración. Suministrados a ratas en período de lactancia, han producido supresión del crecimiento e interferencia con la producción endógena de estas sustancias, a mas de otros efectos indeseables. Estas alteraciones no se han constatado en niños. Con todo, es prudente aconsejar a las madres que reciban dosis terapéuticas de corticosteroides que no amamanten a sus hijos $(25,31)$.

TIROXINA $Y$ TRIYODOTIROXINA endógenas se encuentran en la leche materna en cantidades apreciables; también se han encontrado las exógenas pero en concentraciones muy bajas $(\delta)$.

\section{Narcóticos}

Los opiáceos, en ‘general, se detectan y dosifican en la leche materna. Teóricamente podrían llevar al lactante a la toxicomanía, pero es tan reducida la concentración alcanzada en la leche materna, que parece muy improbable esta posibilidad. Cuando se administran dosis altas se eleva la concentración en la leche materna y por ello se considera la lactancia como un excelente método de tratamiento en la adicción neonatal congénita (13).

HEROINA. Cuando la madre toxicómana ingiere dosis elevadas, se pueden encontrar concentraciones altas en la leche capaces de inducir adicción en el lactante (29).

METADONA (Dolofín). Tomada a dosis terapéutica alcanza a suministrar un promedio de $57 \mathrm{mcg}$. diarios al lactante, dosis considerada como insignificante y sin peligro. Sin embargo Smialek informa la muerte de un lactante cuya madre ingería metadona (44). Es posible evitar al lactante la ingestión de altas concentraciones de metadona administrando a la madre la dosis diaria por la noche después de la última tetada y dando al niño un biberón a la hora de la próxima alimentación.

MORFINA. Cuando se administra a dosis terapéuticas usuales, sólo se detectan mínimas cantidades en la leche materna. 


\section{Sedantes y tranquilizantes}

CLORAL HIDRATO. Se encuentra en la leche materna en concentraciones lo suficientemente altas como para producir sedación en .el lactante (4).

CLORPROMAZINA. (Largactil). Se han encontrado niveles altos en la leche materna, concomitantes con somnolencia del lactante (53).

DIAZEPAN (Valium). Algunos autores afirman que no se excreta por la leche (12). Patrick comunicó el caso de un lactante que presentó letargo y perdió peso progresivamente; la madre tomaba dosis terapéuticas de diazepán (37)

LITIO. El carbonato de litio se emplea cada día más como integrante básico de muchos preparados farmacéuticos ușados en el tratamiento de estados maníaco depresivos. Usado esporádicamente no produce efecto tóxico a niños amamantados por estas madres, pero cuando su administración es prolongada se detectan altas concentraciones que pueden acarrear reacciones perjudiciales tales como hipotonía, hipotermia, cianosis y modificaciones en el electroencefalograma, como sucedió en el caso descrito por Tunnessen en un lactante menor de un año (43, $47,56)$.

\section{Otros medicamentos}

ALCOHOL. Normalmente se aconseja a la madre que amamanta no ingerir alcohol. Esta recomendación tiene como base una observación de Bisdom en 1936: la madre ingirió 750 cc. de vino oporto en 24 horas y el niño presentó sueño profundo del que no podía sacarse, acompañado de ronquidos, respiración profunda, sudoración excesiva, disminución de reflejos y falta de reacción a estímulos dolorosos (6). Sin embargo esta observación no ha podido confirmarse en los 44 años siguientes. La concentración de alcohol en la leche materna es muy similar a la que se encuentra en su sangre. Se desconoce qué cantidad pueda tolerar el lactante en las 24 horas.
Parece que muy a pesar de que haya un alto contenido de alcohol en la leche materna, y el niño reciba una buena cantidad de esta leche, no se aprecian sintomas en el lactante, salvo el caso de madres alcohólicas crónicas exageradas, capaces de llegar a concentraciones alcohólicas sanguíneas muy altas (9). Binkiewics de Boston publicó el caso, muy bien estudiado por cierto, de un niño lactante, de cuatro meses de edad, con un síndrome de seudo-Cushing, hijo de madre que ingería diariamente y de tiempo atrás, dosis muy altas de bebidas alcohólicas (5). Como principio práctico podemos permitir a la mujer que lacta, la ingestión moderada y esporádica de alcohol advirtiendo que la combinación alcohollactancia no es deseable.

ATROPINA. Se pueden encontrar en la leche materna cantidades suficientemente capaces de producir efectos anticolinérgicos en los lactantes, muy sensibles de por sí al fármaco, probablemente por inmadurez de la placa motora neuromuscular (10).

CAFEINA. Se encuentran cantidades detectables en la sangre de niños alimentados al seno, pero por lo general estas concentraciones son demasiado bajas, incapaces de producir efecto farmacológico. Sin embargo, como los niños son más suceptibles que los adultos al estímulo de la cafeína, puede ocasionarles una alta excitabilidad refleja e intranquilidad (41). Esta dosis circulante en la sangre de los niños no siempre es excretada con facilidad.

CLOFIBRATO (Atromid S). No se sabe si se excrete por la leche humana, pero como en experimentación animal se han encontrado en el suero fetal concentraciones más elevadas que en el suero de la madre en época en que es posible que el feto no haya desarrollado el sistema enzimático requerido para la excreción del clofibrato. La casa productora recomienda no tomarlo durante la lactancia.

CORNEZUELO DE CENTENO. Se usan los alcaloides. Se excretan por la leche $y$ se puede afirmar que el $90 \%$ de los 
niños que los ingieren presentan signos de ergotismo cuando se administran a la madre en dosis altas y por tiempo prolongado.

Estos signos son: náuseas, vómito, cólicos abdominales, diarrea, enfriamiento de las extremidades, somnolencia o insomnio, inquietud y aun convulsiones (12).

DIGITALICOS. No se han podido detectar en el plasma de lactantes cuyas madres recibian tratamiento digitálico (30).

LAXANTES. El aceite mineral y los laxantes que aumentan el volumen del residuo no llegan a la leche materna. En cambio los catárticos tales como la cáscara sagrada, el sen, la emodina y la antraquinona pueden producir en el lactante cólico intestinal, diarrea y náuseas $(22,24,45)$.

MARIHUAANA. Su principal componente activo, el tetrahidrocanabinol, es soluble en las grasas y por lo tanto hay posibilidad de que se pueda detectar en la leche materna, pero hasta el presente no se han comunicado efectos indeseables en los lactantes (10).

NICOTINA. No se altera la secreción láctea en aquellas mujeres que fuman moderadamente (38). Las concentraciones de nicotina en leche de madres fumadoras son bajas y parece que guardan relación con el número de cigarrillos fumados en el día. Fuera de un caso reportado por Bisdom en 1936 (niño de seis semanas alimentado al seno, cuya madre fumaba veinte cigarrillos diarios, presentó intranquilidad, vómito, diarrea, taquicardia, trastornos circulatorios e insomnio) (5), no se han descrito efectos adversos en lactantes (18). Se considera como contraindicación formal la lactancia materna cuando la madre fuma más de veinte cigarrillos al día (36).

MEDICAMENTOS CONTRA EL CANCER. CITOSTATICOS. Son muy escasos los datos al respecto. Amanto observó que un lactante cuya madre recibía $\mathrm{Cl}$ CLOFOSFAMIDA hizo depresión de médula ósea (1).

TECTENIO RADIOACTIVO. Se ha encontrado en la leche cuando ha sido administrado para tomografía axial computarizada. Se sugiere suspender la lactancia por dos días después de practicado este examen (57).

\section{Alimentos}

Hay la creencia popular de que ciertos alimentos que se emplean para condimentar o dar sazón a la comida pueden causar al lactante algunas alteraciones del aparato digestivo tales como cólicos, vómito, diarrea. Se culpa principalmente at ajo, a la cebolla, al tomate y al aguacate. No conocemos estudios suficientemente serios que confirmen si éstos $u$ otros alimentos tomados por la madre pueden, dar efectos indeseables al lactante.

\section{Conclusiones}

Hay poca información sobre los efectos perjudiciales que los fármacos administrados a la madre pueden producir en los lactantes, sobre todo cuando ella los recibe a dosis altas y a largo plazo.

Al prescribir cualquier droga a la madre que lacta debe considerarse si el beneficio para la madre justifica el posible riesgo que existe para el bebé.

Las madres tratadas con sustancias antitiroideas, especialmente yodo radiactivo, litio, cloranfenicol, fármacos anticancerosos, antimetabolitos, anticoagulantes, tetraciclina, ergotamina, atropina, metronidazol, tinidazol, no deben amamantar.

Se desconoce la inocuidad de muchas drogas que se administran durante la lactancia.

No hay información seria acerca del efecto que sobre el lactante puedan tener algunos alimentos ingeridos por la madre. 
Durante la lactancia es prudente evitar, en cuanto sea posible, la administración de fármacos a la madre.

\section{DRUGS IN MATERIAL MILK}

\section{Conclusions}

There is few information available on the harmfull effects that the drugs administered to the mother may produce on suckling infants, specially when she receives them at high doses and for a long period of time.

When prescribing any drug to a nursing mother, it must be considered if the benefits to the mother justify the possible risk for the baby.

Mothers treated with antithyroidic substances, particularly radioactive iodine, litium, chloralphenicol, anticancerous drugs, antimethabolites, anticoagulants, tetracicline, ergotamine, athropine, metronidazol and tinidazol, must not nurse.

The harmlessness of many drugs administered during the nursing period is unknown.

There is not reliable information on the effect that many food-stuffs eaten by the mother may have on suckling infants.

During the nursing period, it is adviseable to avoid as much as possible the administration of drugs to the mother.

\section{Bibliografía}

1 AMATO, D. y NIBlETT, J. S., Med. J. Aust. 1: 383, 1977.

2 ANDERSON, P. O. y SALTER, F. J. AM. J. CARDIOL. 37: 325, 1976.

3 BELTON, E. M. y JONES, R. V.Lancet, 2: $691,1935$.
4 BERNSTINE, J. B. y cols. J. Obstet. Gynec. Br. Emp. 63: 228, 1956.

5 BINKIEWICZ, A. y cols. J. Pediatrics. 93: 965, 1978.

6 BISDOM, C. J. W. citado por Catz, Ch. S. en Clín. Ped. de N. Am. 1: 151, 1972.

7 BLAND, E. P. y cols. Lancet, 2: 1039, 1969.

8 BODE, H. H. y cols. Pediatrics. 62: 13 1978.

9 BRAMBEL, E. E. y HUNTER, R. E. Am. J. Obstet. Gynecol. 59: 1153, 1950

10 Carta Médica. 16: 27, 1974 y 21: 26, 1979

11 Carta Médica. 12: 63, 1970.

12 CATZ, Ch. S. y Giacoia, G. P. Clín. Ped. de N. Am. 1: 151, 1972.

13 COBRINIK, R. W. y cols. Pediatrics. 24: 288, 1959.

14 CURTIS, E. M. Obstet. Gynecol. 23: 295, 1964.

15 DUARTE-CONTRERAS, A. y cols. Rev. Colomb. Obst. Gin. 22: 309, 1969.

16 ECKESTEIN, H. B. y JACK, B. Lancet. 1: 672, 1970.

17 EPSTEIN, S. S. Cáncer Res. 34: 2425, 1974.

18 FERGUSON, B. B. y cols. Am. J. Dis. Child. 130: 837, 1976.

19 FOUTS, J. R. y HART, L. G. Am. New York Acad. Sci. 123: 245, 1955.

20 GOODMAN, L. y GILMAN, A. The Pharmacological Basis of Therapeutics, 5 ed. New York, Mac Millan Ed. 1353, 1975.

21 GREEN, H. J. y cols. Am. J. Obstet. Gynec. 51: 732, 1946.

22 GREENHALF, J. O. y cols. Practitioner. 210: 259, 1973.

23 HARLEY, J. D. y ROBIN, H. Pediatrics. 37: 855, 1966.

24 JONES, K. L. y cols. Lancet. 2: 999, 1973.

25 KATZ, F. H. y DUNCAN, B. R. N. Engl. J. Med. 293: 1154, 1975.

26 KNOWLES; J. A. J. Pediat. 66: 1068, 1965.

27 KNOWLES, J. A. Drug. Ther. 3: 57, 1973. 
28 LAUMA, R. K. y cols. Am. J. Obstet. Gynecol. 98: 411, 1964.

29 LICHTENTEIN, P. M. New York State, Med. J. 102: 905, 1915.

30 LODGHNAN, P. M. J. Pediatr. 92: 1019, 1978.

31 McKENZIE, S. A. y cols. Arch. Dis. Child. 50: 894, 1975.

32 MEYERS, F. H. y cols. En Farmacología Clínica, 3 ed. México, Ed. El Manual Moderno, 297, 1977.

33 MIRKIN, B. L. J. Pediatr. 78: 329, 1971.

34 MULLEY, B. A. y cols. Eur. J. Clin. Pharmacol. 13: 129, 1978.

35 NIEBLY, J. R. y cols. Obstet. Gynecol. 53: $139,1979$.

36 OSEID, B. J. Clin. Obst. Gin. N. Am. 2: 143, 1975.

37 PATRICK, M. J. y cols. Lancet. 5: 542, 1972.

38 PERLMAN, H. H. y col. JAMA, 120: 1003, 1942.

39 PINCUS, G. y cols. Nature (London) 212: 924, 1966.

40 RISTIA, M. y SHUBIK, K. F. J. Nat. Can. Inst. 48: 721, 1972.

41 RIVERA-CALIMLIM, L. Drug. Ther. 7: 59, 1977.

42 ROllieR, M. R. y col. Bull. Soc. Franc. Dermatol. Syph. 74: 178, 1967.
43 SCHOU, M. y AMDISEN, A. Br. Med. J. 2: $138,1973$.

44 SMIALEK, J. E. y cols. JAMA. 238: 1516, 1977.

45 StONE, S. C. y col. Clin. Obst. Gin. N. Am. 2: 133, 1975.

46 YAKYI, B. E. J. Hosp. Pharmacy. 28: 317, 1976.

47 TUNNESSEN, W. W. y HERTZ, C. G. J. Pediatr. 81: 804, 1972.

48 TYSON, M. y col. J. Pediatr. 13: 86, 1938.

49 VERSANO, I. y cols. J. Pediatr. 82: 886, 1973.

50 VOOGD, C. E. y cols. Mutat Res. 26 483, 1974.

51 WALKER, W. A. y HONG, R. J. Pediatr. 83: 517. 1973.

52 WERTHMANN, M. M. y KREES, S. V. J. Pediatr. 81: 781, 1972.

53 WILES, D. H. y cols. Br. J. Clin. Pharmacol. 5: 272, 1978.

54 WILLIAMS, M. Pharmaceut. J. 217: 219, 1976.

55 WILliAMS, R. A. y cols. J. Clin. Invest. 23: $613,1944$.

56 WOODI, J. N. y cols. J. Pediatr. 47: 94, 1971.

57 WYBURN, J. J. Nucl. Med. 14: 115, 1973.

58 YURCHAK, A. M. y JUSKO, W. J. Pediatrics. 57: 518, 1976. 